\title{
Effect of Evaporation on the Growth Kinetics in a Model for Two Species
}

\author{
Hassan F. El-Nashar \\ Physics Department, Faculty of Science, Ain Shams University, \\ Cairo, Egypt
}

A surface growth model for two species is proposed, when deposition, surface diffusion and evaporation are considered, in (1+1)-dimensions. A Monte-Carlo simulation is carried out, focusing on the effect of evaporation on the evolution of the amount of roughness. The results show that the interplay between deposition, surface diffusion and evaporation slows down the rate of growth of the surface width. In addition, when the rate of evaporation increases, the surface width grows faster to a higher value, in comparison to the case of low rate of evaporation. This introduces changes in the scaling exponents which show that evaporation should be given equal or as much consideration as deposition and surface relaxation.

\section{Introduction:}

The growth of surfaces attracts interest due to its technological importance since many properties of materials depend on surface roughness. During the growth, the surface becomes undesirably rough. In order to avoid this morphology, the basic physical effects and the processes that lead to the development of surface roughness must be well understood. Apart from specific technological applications, most rough surfaces are formed under conditions that are far from equilibrium. Therefore, the study of roughening has a relevant importance in the understanding of nonequilibrium statistical physics at the fundamental level [1-3]. It is well known that a stochastic growing surface exhibits scaling behaviour and evolves to a steady state without a characteristic time or length scale. This has led to the development of the dynamical scaling approach which has been found by Family and Viscek [1]. Starting with a flat substrate, defining the surface width $W(L, t)$ as

$$
W^{2}(L, t)=\frac{1}{L^{d-1}} \sum_{r}[h(r, t)-\overline{h(t)}]^{2}
$$


where $L$ is the system size, $h(r, t)$ is the height of the surface at position $r$ and time $t, d-1$ is the substrate dimension and $\overline{h(t)}$ is the average of the surface height, the scaling law takes the form

$$
W(L, t)=L^{\alpha} f\left(t / L^{z}\right)
$$

The dynamical scaling behaviour is characterized by the roughness exponent $\alpha$ and the dynamical exponent $z$ with growth exponent $\beta=\alpha / z$. The function $f(x)$ scales as $f(x)=x^{\beta}$ for $x<<1$ and $f(x)=$ const. for $x>>1$. This scaling behaviour has been studied and has been argued to be universal $[2,3]$.

Discrete growth models have played a major role in the understanding of the nature of the surface roughness since they mimic the essential physical parameters and eliminate details which are less important $[1,3]$. The growth of two or more species is common in modern technology [4]. In this case it is desirable to study aspects such as kinetics and scaling behaviour in order to get microscopic understanding of the growth of the deposition of multi-species. In this situation the dynamics of both types of species is important as well as the processes, such as deposition, surface diffusion and incorporation, etc., which increases the level of difficulty of the problem, to consider all of them at once. The use of a rather simple model for two species which includes only simple processes, will give insight into the dynamics of roughening. It is common in vapour deposition to find local diffusion of the newly arrived particles along the surface of the deposited material. Even though the models regarding the growth were designed to include deposition and surface relaxations [1-3], there could also be evaporation processes in the real growth. Therefore, in order to see only the effect of evaporation on the growth kinetics, there should be a simple model in which we are able to focus on the evaporation processes during the growth. Henceforward, one can get answers to the following questions: "does evaporation affect the kinetic roughening? and is it an important process to be considered in the case of surface growth of two species?".

Few models for two species have been introduced which incorporate evaporation into surface growth models [5, 6]. In reference [5], the authors studied the effect of the rules of interaction between the different species on the universality. In reference [6] a model for two species (active and inactive) was introduced which allows surface diffusion and evaporation. Such a model shows growth kinetics where the values of exponents differ from the values of the Edwards-Wilkinson's (EW) [7] universality, although it is a random deposition model with diffusion (RDD) [1-3]. However, in the previous work, the effect of evaporation processes on growth kinetics was not resolved from other processes. In order to resolve only the evaporation from other processes, we 
need to fix the rate at which these other processes occur. Then, the growth kinetics can be investigated in order to test the effect of the evaporation on the kinetic behaviour.

\section{Model:}

In this work, a random deposition model with diffusion for two species is proposed, in which evaporation is allowed to take place in such a way, that this is the only process under control. We do not allow overhang/voids to occur during the growth in order to keep the model simple. The model represents the surface growth due to the deposition of two kinds of species with different attractive forces where the temperature is sufficiently high such that the less active particle is allowed to evaporate. The model does not describe the growth of a specific material, rather it concentrates on the effect of evaporation in the case when the dynamics of both species is considered. In order to model the growth of real material, e.g, semiconductors [4], some details should be included which will make the model complicated, thus testing only the effect of evaporation will be difficult.

In general, for the model under study here, the rules of interaction are such that one type of species (A), active, is always necessary for the deposition of both species to occur, while the other (B), less active than (A), is allowed to evaporate or to control the diffusion across the surface. Therefore, evaporation and surface diffusion will depend on the concentration of the (B) species. In this case these processes interfere with each other and the growth occurs according to the interplay between both of them, in addition to the deposition. Since surface diffusion and evaporation depend on the presence of particles (B), we will fix the rate of surface diffusion and will increase the rate of evaporation, where the concentration of both species remains same. This will be clear below when we describe the model. We believe that the increase in the rate of evaporation will produce a change in the topography of the surface during the growth. This change can be characterized by the evolution of the amount of roughness (kinetic roughening).

The growth occurs as follows: at first a site $j$ is selected at random and then a particle (A) (or particle (B)) is deposited on the surface with a probability 1-P (or $P$ ); i.e, the deposition rate of each particle depends on the chosen probability $P$. Fig. 1(a) shows a piece of the aggregate for model (i) and Fig. (1b) represents that of model (ii). The white squares represent the aggregated particles of type (A) and dark squares represent those of type (B). Circles denote the incoming particles while the down arrows represent deposition. The shifted down arrows represent diffusion and the inclined dashed arrows indicate evaporation. As shown in Fig. (1a), if the arriving particle is of type (A), it is 
allowed to deposit and stick over a particle if it is of the same type (particles 2, 3,4 and 7) otherwise, it diffuses to a local minimum if the deposition occurs over (B) type (particles 1 and 6). For a process 5, deposition occurs for (A) type and there is no diffusion since this site represents a local minimum. If the incomming particle is of type (B), it is allowed to deposit over type (A) and then diffuses to a local minimum (particles 2, 3, 4 and 7). The evaporation occurs for (B) type as shown for processes 1 and 6 . The deposition of (B) type, which is indicated by 5 , occurs due to a presence of (A) type in a neighbouring column and higher by one step. The modification of model (i) in order to increase the rate of evaporation is shown in figure 1(b) which represents model (ii). After deposition of (B) type over (A) type, diffusion to a local minimum occurs. If this diffusive particle ends over a (B) type, where there is no (A) type in any neighbouring site higher by one step, then it evaporates (particles 3 and 4). This modification increases the rate of evaporation for the same value of the deposition probability $P$.

\section{Results:}

The simulation is performed on a lattice of size $L$ with a periodic boundary conditions such that the chosen site $j$ is the same as $j+L$. Statistical average is obtained over 200 independent simulations for each parameter. The simulation is carried out on the computer by means of a Monte-Carlo method. In order to simulate the processes according to models (i) and (ii), a computer program is written using the FORTRAN language. We use different random number generators from CERN and EMSSL libraries as well as a code written by the author in order to satisfy the following conditions: a) the random numbers are uniformly distributed in the interval [0-1] and $b$ ) these random numbers are uncorrelated. Throughout the code of the computer program, a two-dimensions array is used to represent the embedded dimensions $d$. The first dimension of the array represents the substrate of dimensions $d-1$ and the second dimension is used for the height of the aggregation. The site on the substrate of location $(j)$ is chosen randomly according to a specific model and then it is filled by a particle of type (A) or (B) according to the value of $P$ or $(1-P)$. The deposition, diffusion or evaporation processes are determined according to the content of the surrounding sites of the newly filled particle in the location ( $j$ ). The time is computed as one Monte-Carlo step. At each MonteCarlo step, the height $h(r, t)$ at each site and the average height $\overline{h(t)}$ of all aggregation are calculated in order to get finally the value of the surface width $W(L, t)$ at this time.
12
34
5
$\begin{array}{ll}6 & 7\end{array}$
O 0
O O
O
O 0 


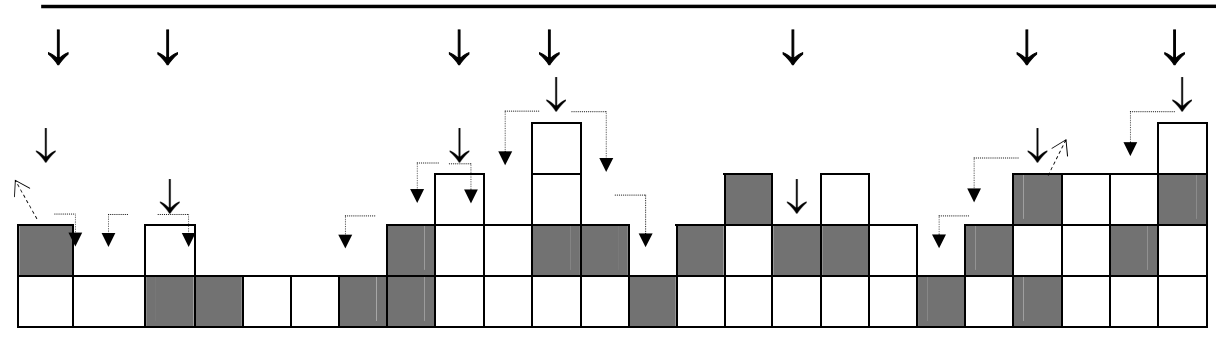

(a) model (i)

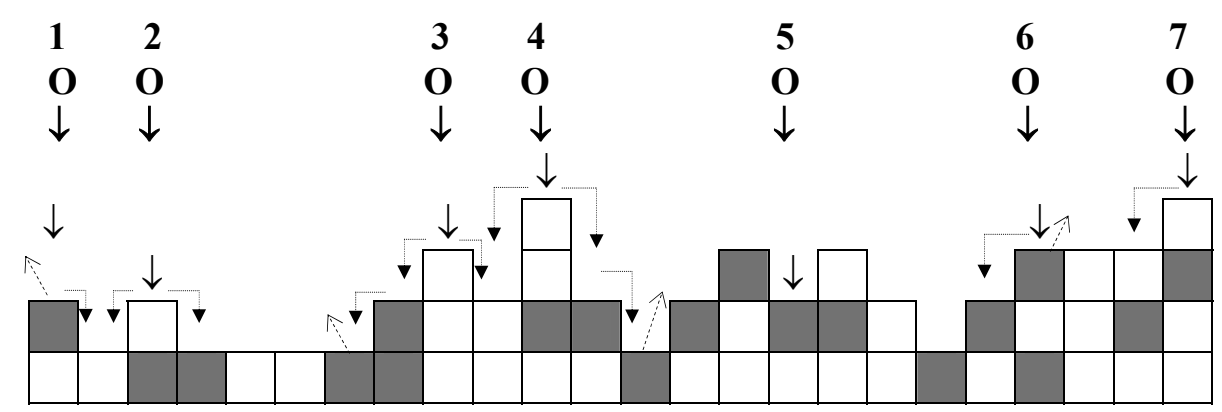

(b) model (ii)

Fig. (1): A piece of the cross section of the aggregate according to: (a) model (i) and (b) model (ii).

The results for model (i) are shown in Fig. (2). In this figure we present a log-log plot of the surface width $W$ as a function of time for three different values of deposition probability $P$ and for RDD model of one type of species [when $P=0$, which is taken as a reference for a comparison]. We see that the surface width decreases as $P$ increases. The inset of Fig. (2) shows a log-log plot of the size $L$ versus the saturated surface width $W_{S}$ from where the values of the exponent $\alpha$ in cases of RDD and $P=0.5$, respectively, are obtained. 


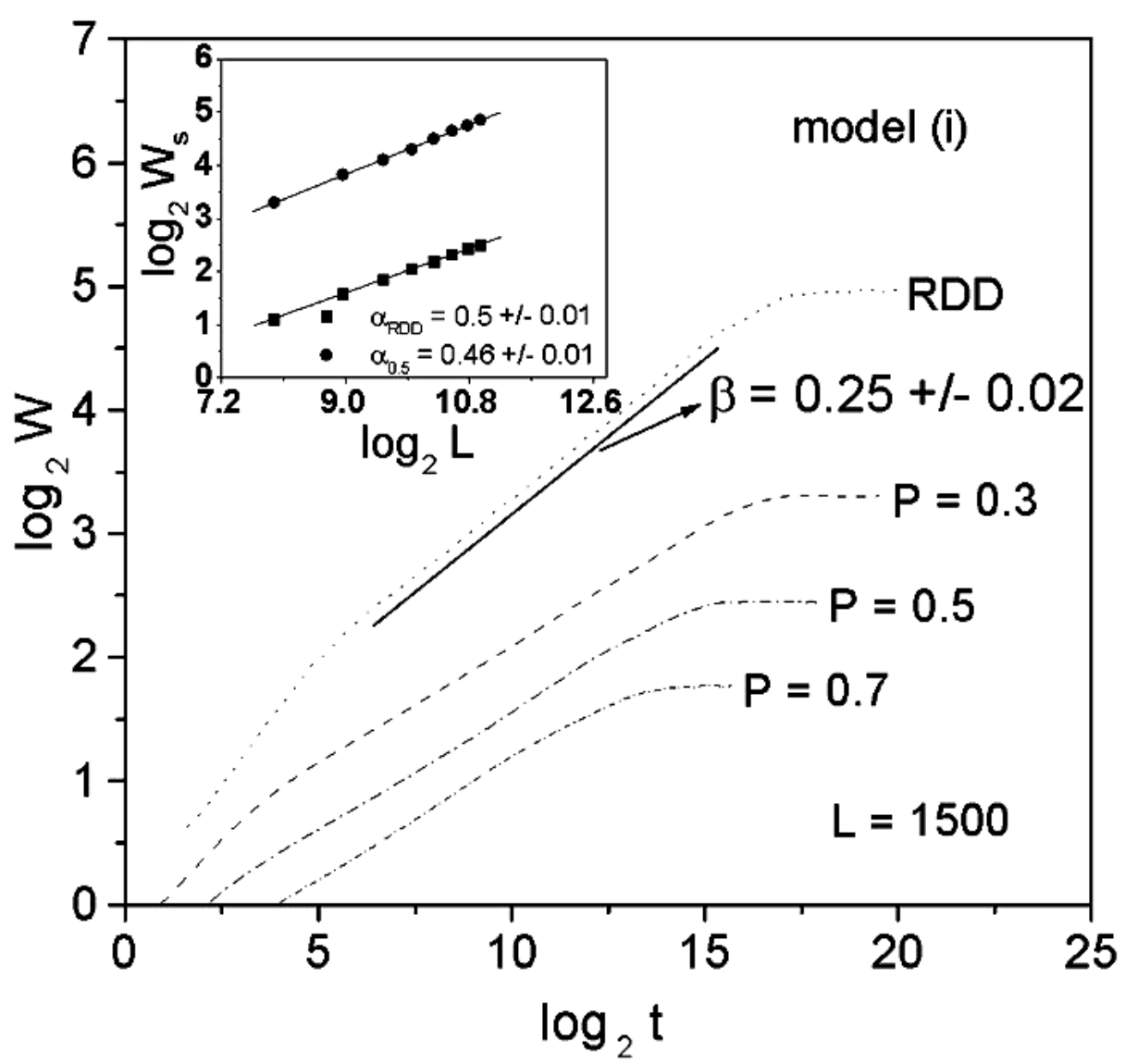

Fig. (2): Log-log plot of the surface width versus time for different $P$ for $\mathrm{L}=1500$ for model (i). The upper inset shows the extraction of the exponents for RDD-model and $\mathrm{P}=0.5$.

Table (1) lists the measured values of the exponents for model (i), which shows that as $P$ increases, the values of the exponents decrease. This means that there is a slow down in the kinetics compared to the RDD model. Therefore, the interplay between deposition, diffusion on surface and evaporation slows down the growth of the amount of roughness and finally it saturates at a lower value when compared with RDD model. As indicated from the model, when $P$ increases, the rates of diffusion and evaporation increase and at the same time the rate of deposition upon first touch to the surface decreases (when (A) type sticks to its same kind). Thus, when the rates of surface diffusion and evaporation increase, the surface width grows more slowly and saturates at a lower value. 
Table (1): The computed exponents for model (i) and for different values of $P$.

\begin{tabular}{|c|c|c|}
\hline $\mathbf{P}$ & $\boldsymbol{\alpha} \pm \mathbf{0 . 0 1}$ & $\boldsymbol{\beta} \pm \mathbf{0 . 0 1}$ \\
\hline \hline 0.3 & 0.48 & 0.2 \\
\hline 0.5 & 0.46 & 0.19 \\
\hline 0.7 & 0.44 & 0.18 \\
\hline
\end{tabular}

However, we can not resolve the effect of evaporation process on the growth kinetics, neither on the exponents from the behaviour shown in Fig. (2). In order to see this effect, we make the log-log plot of surface width as a function of time for different values of $P$ for model (ii) in figure 3 . It is seen from this figure that the surface width decreases as $P$ increases. At the same time, the surface width saturates at a higher value than that of model (i) for each $P$ but still lower than that of the RDD model. Table (2) lists the values of the exponents in the case of model (ii). It is seen from table 2 that the growth exponent $\beta$ remains approximately the same for each $P$ while the exponent $\alpha$ decreases as $P$ increases.

Table (2): The computed exponents for model (ii) and for different values of $P$.

\begin{tabular}{|c||c||c|}
\hline $\mathbf{P}$ & $\boldsymbol{\alpha} \pm \mathbf{0 . 0 1}$ & $\boldsymbol{\beta} \pm \mathbf{0 . 0 1}$ \\
\hline 0.3 & 0.47 & 0.22 \\
\hline 0.5 & 0.44 & 0.22 \\
\hline 0.7 & 0.40 & 0.23 \\
\hline
\end{tabular}

The comparison between the results of model (i) (figure 2) and model (ii) (figure 3) indicates that the surface width, at the same value of $P$ when the rate of evaporation increases (model (ii)), saturates at a higher value than that of model (i). At the same time the growth rate of the surface width is higher in the case of model (ii) than that of model (i). This is clear from table 1 and table 2 when growth exponents in both models are compared for each value of $P$. It is also seen that in the case of model (ii), the growth exponent is closer to the value of that of RDD model (EW universality). However, when evaporation rate increases, the roughness exponent decrease more for model (ii) than that of model (i) while this exponent for both models is lower than that of RDD model. Therefore, we argue that the evaporation process, when it occurs at a low rate, slows down the growth of the surface width. In contrast, when evaporation rate increases, the surface width grows fast in comparison to the low rate but still with a rate lower than that of the RDD model. Finally, the increase in the rate of evaporation leads to a more jagged surface. The lower inset of Fig. (3) shows the log-log plot of the surface width as a function of time for models (i) and (ii) when $P=0.5$ in addition to the RDD model for a comparison. 


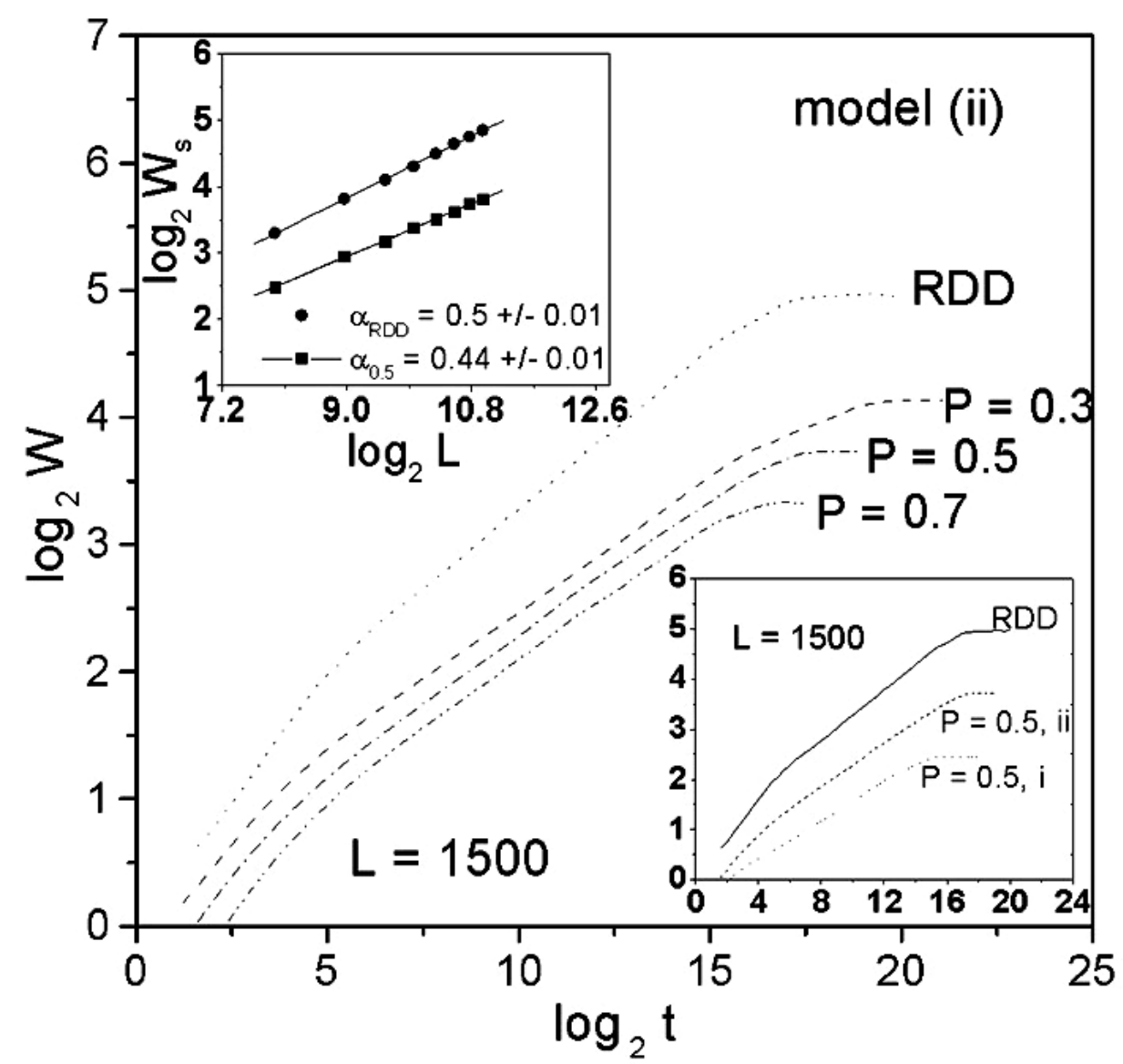

Fig. (3): Log-log plot of the surface width versus time for different $P$ for $L=1500$ for model (ii). The upper inset shows the extraction of the exponents for RDDmodel and $\mathrm{P}=0.5$. The lower inset shows the log-log plot of the surface width for RDD-model, model (i) and model (ii) for comparison.

\section{Conclusion:}

In conclusion, we considered evaporation in addition to deposition and surface diffusion in the RDD model for two assymmetric species. We have seen that the interplay between deposition, surface diffusion and evaporation leads to a different kinetic behaviour and different roughening. The values of the exponents measured in our simulations are deviated from the values of the EW universality of the usual RDD model of one type of species. It is shown that the evaporation process alone has affected the evolution of the amount of roughness which appeared in the behaviour of the surface width $W(t)$ and the saturated 
surface width $W_{S}$. We also show that the increase of the rate of evaporation enhances the growth of the surface width which is indicated from the values of the exponents $\beta$ for different deposition probabilities. It is indicated from the values of the exponents $\alpha$ that the surface becomes rougher when the rate of evaporation increases. In reference [8], RDD model for one kind of species is considered to include desorption. The results of this work show that desorption has no effect on the universality and hence it has no importance to be considered. However, the results of this work show that introducing evaporation in the growth of binary species changes the scaling exponents. Therefore, it should be treated on the same level of importance as well as deposition and surface relaxation.

\section{Acknowledgement:}

HFE would like to thank the MPIPKS, Dresden, Germany for support during some part of this work.

\section{References:}

1. Family F. and Vicsek T., "Dynamics of Fractal Surfaces", World Scientific, Singapore, (1990).

2. H-Healy T. and Zhang Y.-C., Phys. Rep. 254, 215 (1995).

3. Barabasi A.-L. and Stanley H., "Fractals Concepts in Surface Growth", Cambridge University Press, Cambridge, (1995).

4. Politi P., Grenet G., Marthy A., Ponchet A. and Villain J. Phys. Rep. 324, 271 (2000).

5. Park S. and Kahng B., Phys. Rev. E., 60, 6160 (1999).

6. Wang W. and Cerdeira H., Phys. Rev. E., 52, 6308 (1995).

7. Edwards S. F. and Wilkinson D. R., Proc. R. Soc. London, Ser. A 381, 17 (1982).

8. Raychaudhuri S., Shapir Y., Foster D. G. and Jorne J. Phys. Rev. E., 64, 051604 (2001). 